\title{
Span Restoration in Optical Networks with Limited Wavelength Conversion
}

\author{
Ruepp, Sarah Renée; Buron, Jakob Due; Andriolli, N; Wessing, Henrik
}

Published in:

Second International Conference on Communications and Networking in China, 2007. CHINACOM '07.

Link to article, DOI:

10.1109/CHINACOM.2007.4469432

Publication date:

2007

Document Version

Publisher's PDF, also known as Version of record

Link back to DTU Orbit

Citation (APA):

Ruepp, S. R., Buron, J. D., Andriolli, N., \& Wessing, H. (2007). Span Restoration in Optical Networks with Limited Wavelength Conversion. In Second International Conference on Communications and Networking in China, 2007. CHINACOM '07. IEEE. https://doi.org/10.1109/CHINACOM.2007.4469432

\section{General rights}

Copyright and moral rights for the publications made accessible in the public portal are retained by the authors and/or other copyright owners and it is a condition of accessing publications that users recognise and abide by the legal requirements associated with these rights.

- Users may download and print one copy of any publication from the public portal for the purpose of private study or research.

- You may not further distribute the material or use it for any profit-making activity or commercial gain

- You may freely distribute the URL identifying the publication in the public portal

If you believe that this document breaches copyright please contact us providing details, and we will remove access to the work immediately and investigate your claim. 


\title{
Span Restoration in Optical Networks with Limited Wavelength Conversion
}

\author{
Sarah Ruepp*, Jakob Buron*, Nicola Andriolli ${ }^{\dagger}$, and Henrik Wessing* \\ ${ }^{*}$ COM.DTU, Technical University of Denmark, Kgs. Lyngby, Denmark; e-mail: \{sr, jbu, hw\}@com.dtu.dk \\ †Scuola Superiore Sant’Anna, Pisa, Italy; e-mail: nick@sssup.it
}

\begin{abstract}
Next generation optical networks provide functionalities to dynamically provision and recover connections, while emerging technologies allow for the conversion between wavelengths. These devices are however expensive and hence it is likely that only few are deployed throughout the network. Accordingly, the unavailability of wavelength converters decreases the chance for successful connection establishment, especially in the recovery phase, where several restoration requests try to access a limited pool of resources.

The recently proposed Suggested Vector scheme has been designed to perform converter-saving wavelength assignment in GMPLS networks. The converter saving property of the Suggested Vector is particularly desirable in span restoration, where the pre-failure path stubs have to be merged to the restoration path at the failure-adjacent nodes. In order to avoid wavelength conversion at the merging nodes, the wavelength of the connection stubs can be considered.

In this study, we evaluate the recovery performance of the Suggested Vector scheme and a scheme exploiting standard protocol extensions only. The behavior of both schemes with a stub-aware extension for span restoration is also assessed.

Our simulations show that the Suggested Vector scheme achieves a higher recovery performance than the standard scheme. Both schemes benefit from the stub-aware modification. The performance increase is especially predominant if few wavelength converters are available and at medium load ranges.

Furthermore, we describe different scenarios to extend the Suggested Vector wavelength assignment scheme to multi-domain networks with focus on span restoration.
\end{abstract}

\section{INTRODUCTION}

Optical networks are prone to failures. Especially fiber cuts due to construction work are an often recurring problem. A well-known method to recover from a fiber cut is span restoration, where failure affected connections are restored between the failure-adjacent nodes, resulting in a short notification time [1]. In order to setup or restore a connection in an optical network, a route and a wavelength are required. Often, the route is identified first, and then a suitable wavelength is found on the chosen route [2]. Especially in a dynamic network environment, it is important that connections can be setup and restored dynamically. The Generalized Multi-Protocol Label Switching (GMPLS) control plane incorporates these functions [3], using the Link Management Protocol (LMP), the Open Shortest Path First (OSPF) protocol for routing and the Resource Reservation Protocol (RSVP) for resource reservation, the latter two with Traffic Engineering (TE) extensions. The framework of Automatically Switched Optical Networks (ASON) provides a framework to interconnect several GMPLS domains [4].
In a wavelength-routed network, the traffic is switched at wavelength granularity, meaning that each wavelength corresponds to a label in the GMPLS paradigm. The wavelength continuity constraint states that a connection (called Label Switched Path (LSP) in the GMPLS framework) must use the same wavelength on all spans that it traverses from the source to the destination node. This constraint has been relaxed by recent technologies, which allow for the conversion between wavelengths [5]. Wavelength converters (WCs) however are costly devices which means that their deployment throughout the network often is limited, which can lead to connection blocking [6]. It it therefore imperative, that LSPs are provisioned in a WC-saving way. In [7], a provisioning scheme exploiting the novel Suggested Vector (SV), that allows ranking of different wavelengths (i.e. labels) depending on their WC usage was proposed. The SV object works together with the Label Set [8], which is a standard protocol extension and contains the labels that are available on a span. Applying the SV helps saving WCs because they are only used when really needed, i.e. when resources are available, but no wavelengthcontinuous path can be found.

WCs are especially necessary during the restoration phase because many restoration requests try to gain access to limited resources right after the failure [9]. Furthermore in span restoration the restoration path needs to be merged to the prefailure path's stubs at the failure-adjacent nodes, as illustrated in fig. 1. This implies that if the stub label is different from the restoration path label, a WC must be utilized at the failureadjacent nodes.

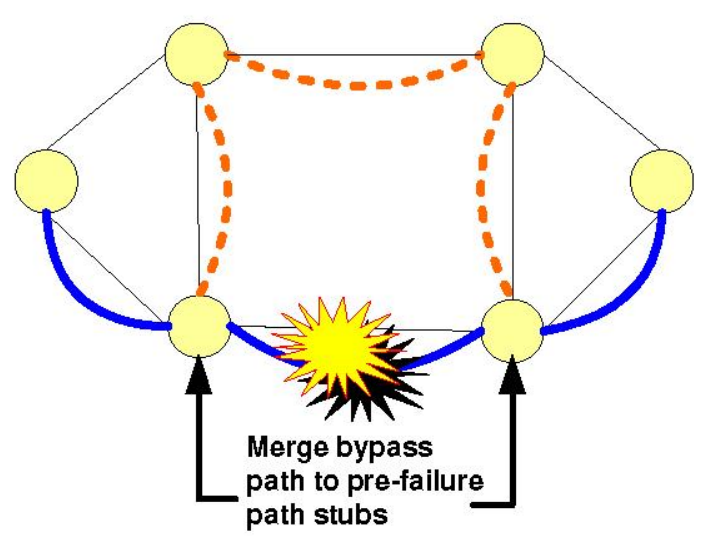

Fig. 1. Span restoration concept. 
A stub-aware scheme based on the SV that takes the label of the original pre-failure path at the failure-adjacent nodes into account when provisioning the restoration path was proposed in [10], and was used to evaluate the usage of WCs per recovered connection in a scenario with an unlimited number of WCs available per node compared to a simple benchmark scheme. In this study, we limit the number of WCs per node and evaluate how this limitation affects the recovery percentage for span restoration of single span failures, when multiple recovery retries are allowed. Furthermore, we extend the stub-aware concept for the standard wavelength assignment scheme, based on the Label Set object. Four scenarios are studied, resulting from the combination of Label Set or SV wavelength assignment together with stub-awareness or stubunawareness. In addition, we broaden the scope of the SV to include restoration in multi-domain networks.

The remainder of this paper is organized as follows: section II explains the operation of the stub-aware label assignment scheme. Section III presents the simulation study and section IV illustrates the results. In section V, the concept of using the SV to reduce wavelength conversion is extended to multi-domain networks. Section VI concludes the paper.

\section{Stub-Aware Label Assignment}

When a route has been found using the OSPF-TE proto$\mathrm{col}$, the RSVP-TE protocol is used to reserve the necessary resources on that route. This is done by propagating Path and Resv messages between the LSP end points. The Path message is used to request a resource, and it may contain optional objects such as the Label Set [8] or the SV [7]. The SV-value for a given label is increased by one, if it requires a WC at an intermediate node. When the Path message has reached the destination node, it chooses the label requiring fewest WCs and issues a Resv message to reserve the desired label. The Resv messages are propagated along the route and a suitable label is chosen at each hop until the source node is reached, which concludes the setup of an LSP. The concept of setting up an LSP with the SV is illustrated in fig. 2.

When setting up the restoration path, the label assignment schemes, i.e. Label Set and SV, can either disregard (stubunaware) or use (stub-aware) the information about the stubs of the failed connection. In the stub-unaware schemes, no

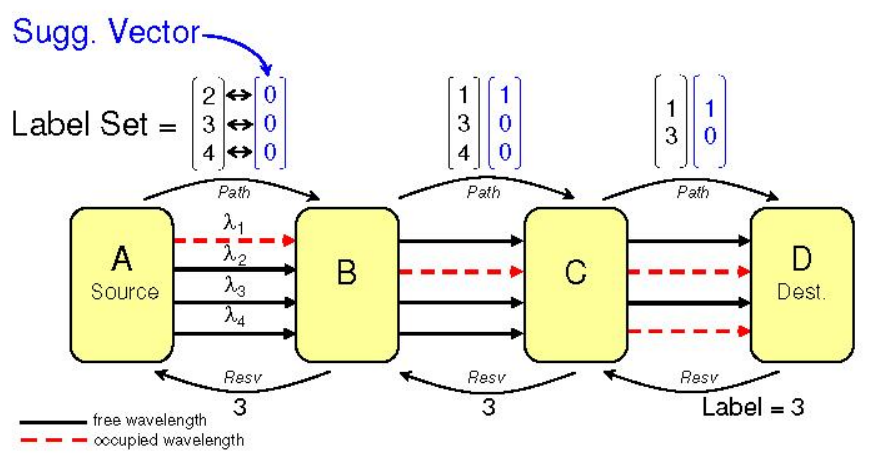

Fig. 2. Path setup with the Suggested Vector (SV) scheme.

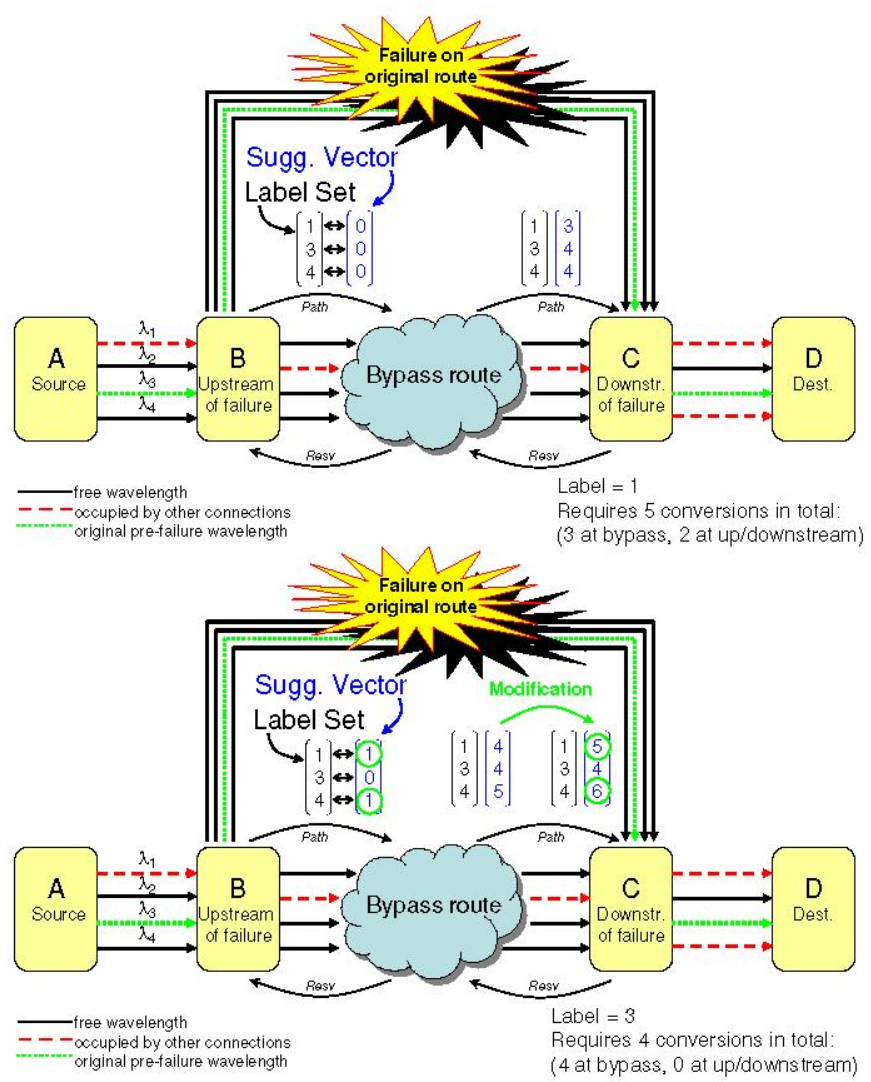

Fig. 3. Suggested Vector application in span restoration. Top: stub-unaware scheme, Bottom: stub-aware scheme with modifications circled

stub information is used and the span restoration is performed exactly as if a new LSP was being setup between the two failure-adjacent nodes without considering that the stubs of the original LSP were assigned to a specific label. In the case of stub-aware schemes, stub information is used in the label selection.

When executing span restoration with the stub-unaware schemes, all labels are given equal preference at the node initiating the restoration path. Similarly, the destination node does not consider the stubs when choosing the label. Hence, in case the SV scheme is used, the SV-value only contains the number of WCs used on the bypass route. This means that the potential conversions to the LSP stubs at the failure-adjacent nodes are not considered, which may lead to a waste of WCs and hence lower restorability. When using both SV and Label Set in stub-unaware mode, all free labels are included in the Label Set. In the special case of 0 available WCs in the upstream failure adjacent node, the stub-unaware scheme has the unwanted behavior of adding all available labels on the next link to the label set, although only the pre-failure label can be used.

The stub-aware label assignment schemes exploit all information about the pre-failure path in the restoration process. Specifically, the label of the pre-failure path's stubs is taken into account when ranking the labels and those that cannot be used due to lack of WCs in the failure-adjacent nodes 
are disregarded completely. This extension avoids unnecessary wavelength-conversion at the failure-adjacent nodes. We hence modify the SV scheme by giving the non-stub labels a value of 1 , as their choice requires a WC at the upstream failureadjacent node. The stub label keeps its SV-value of 0 , since its choice will require no conversion when merging the recovery path to the original path. At the downstream failure-adjacent node accordingly, the SV is examined and modified by adding 1 to all labels except the stub label, since their choice induces a WC. The SV-values now represent the necessary WCs at the upstream failure-adjacent node, the bypass route and the downstream failure-adjacent node. Afterwards, the label with the lowest SV-value is chosen and propagated in the Resv message. The concept of the SV and the stub-aware $\mathrm{SV}$ is illustrated in fig. 3 . The stub-aware scheme has the advantage that only the failure-adjacent nodes have to modify their behavior, whereas no change of algorithm is required at intermediate nodes on the restoration path. The modification to the stub-aware Label Set scheme is to disregard unusable labels when there are no free WCs in the upstream failure adjacent node.

\section{Simulation Study}

In this study, we investigate how the number of WCs per node and the network load affect the recovery percentage when using the Label Set scheme, i.e. the best performing scheme utilizing standard GMPLS signaling, and the SV scheme, both with and without stub-awareness in span restoration. If several equally good labels can be chosen, we use a first-fit tie breaking policy [2].

We evaluate the schemes' performance in the NSFNET [11], illustrated in fig. 4, using OPNET Modeler [12]. The network is populated with unidirectional LSPs based on a uniform distribution of the source/destination pairs until a specified average span load is reached. The chosen label assignment scheme is used in both the provisioning and restoration phase. The Label Set scenario represents the case where the network nodes supports only standard RSVP-TE protocol objects, and hence the Label Set is used for both provisioning and restoration. In the SV-enabled scenario, it is realistically assumed that all nodes support the SV optional object, and hence the SV is used for both provisioning and restoration. In the stub-aware scenarios, the Label Set or the SV are respectively used in the provisioning phase, since stub-awareness is only relevant during the recovery phase. An overview of the different simulation scenarios can be seen in table I.

TAB LE I

SIMULATION SCENARIOS

\begin{tabular}{|c|c|c|}
\hline Simulation scenario & Provisioning scheme & Restoration scheme \\
\hline Label Set & Label Set & Label Set \\
Stub-aware Label Set & Label Set & Stub-aware Label Set \\
SV & SV & SV \\
Stub-aware SV & SV & Stub-aware SV \\
\hline
\end{tabular}

Once the desired load is reached, a span is failed and recovery is attempted. If the recovery request experiences

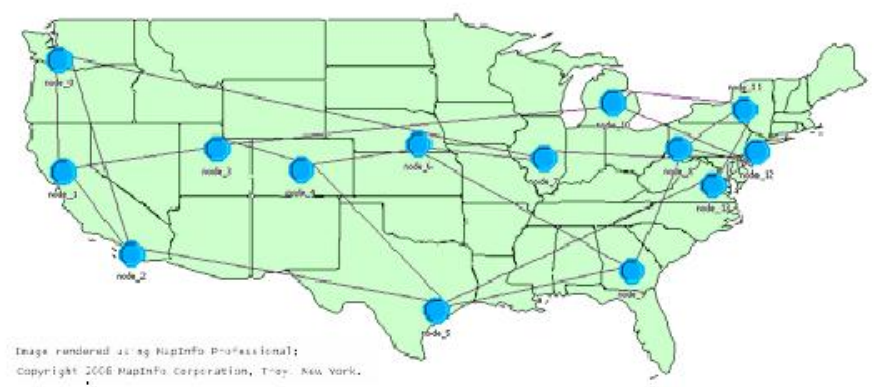

Fig. 4. NSFNET used in simulation study.

blocking, the blocking-causing span is temporarily removed from the topology database using the experimental crankback feature [13], and restoration is reattempted after a backoff period. When all LSPs are either restored or found unrecoverable, the network is reversed to its pre-failure state before the next span failure is evaluated. This procedure continues until the failure of all spans in the network has been simulated.

\section{RESULTS}

All results are obtained by averaging 20 repetitions with different random seeds. The recovery percentage is calculated as the ratio between the number of recovered LSPs and the number of failed LSPs for each span failure, averaged over all span failures. The error bars show the confidence intervals at $95 \%$ confidence level. Fig. 5 illustrates the recovery percentage obtained with a varying number of WCs per node at a constant average span load of 0.5 . If no WCs are available, the Label Set and the SV perform the same, whereas the stub-aware extension for both schemes achieves a better performance, since the label set is restricted to contain only the label that ensures a wavelength-continuous path on the bypass route and the connection stubs, hence avoiding potential merging issues at the failure-adjacent nodes. When more WCs are available, a general tendency to observe is that the recovery percentage increases. Also, the SV outperforms the Label Set in terms of recovery percentage. The advantage of the stub-

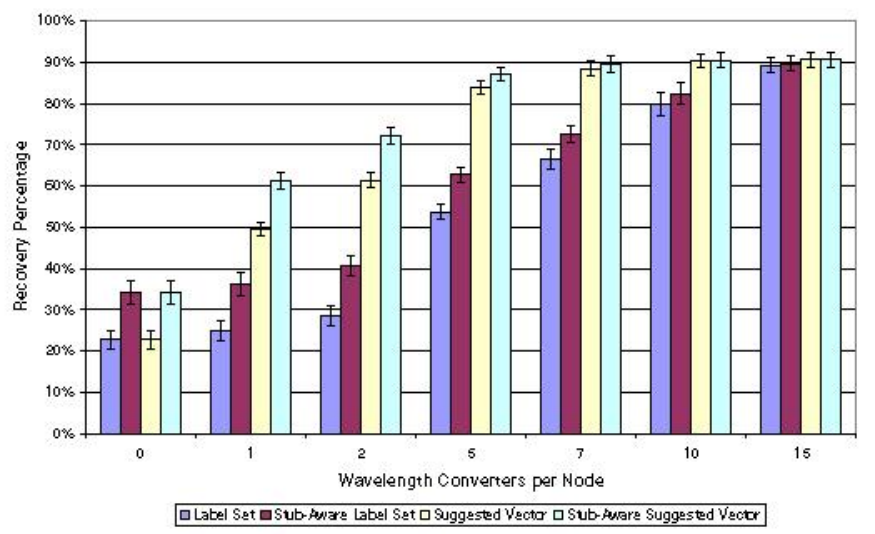

Fig. 5. Recovery percentage with varying number of WCs per node. Average span load is fixed at 0.5 . 


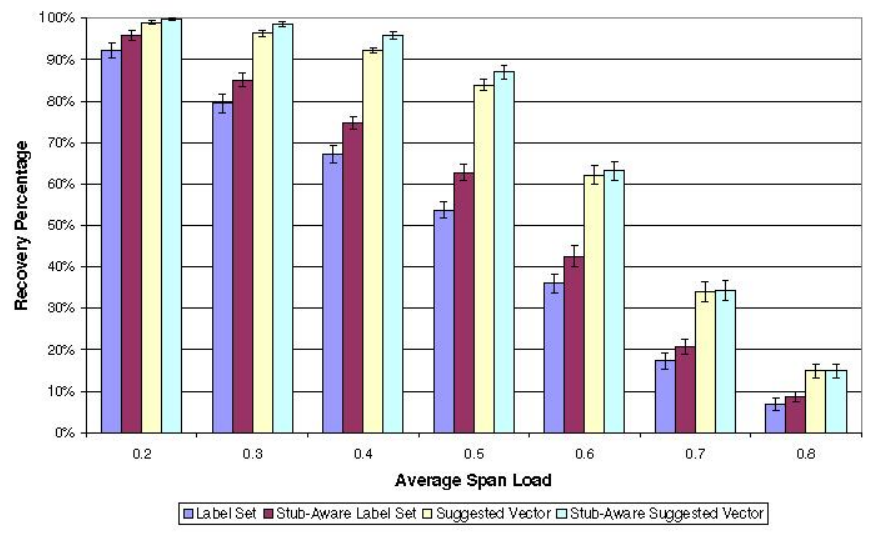

Fig. 6. Recovery percentage with varying average span load. 5 WCs per node.

aware modification for both the Label Set and the SV is especially predominant if WCs are allocated very sparsely, i.e. 5 or fewer WCs per node. However if many WCs are available they are no longer the limiting factor, and the difference between the schemes diminishes.

Fig. 6 shows how the recovery percentage for the different schemes changes with a varying average span load. There are 5 WCs available per node. As expected, the recovery percentage decreases when the average span load is increasing, because fewer spare resources are available for recovery purposes. The stub-aware modification gives the highest performance improvement (compared to the stub-unaware case) at medium load ranges, where the WCs are the limiting resource for restoration. Also, in these conditions the performance increase of the SV compared to the Label Set is highest.

\section{Application to Multi-Domain Networks}

In this section, some considerations are provided for extending the SV scheme to a multi-domain environment with heterogeneous technologies and administrative policies. Such an environment is shown in fig. 7, where the interfaces

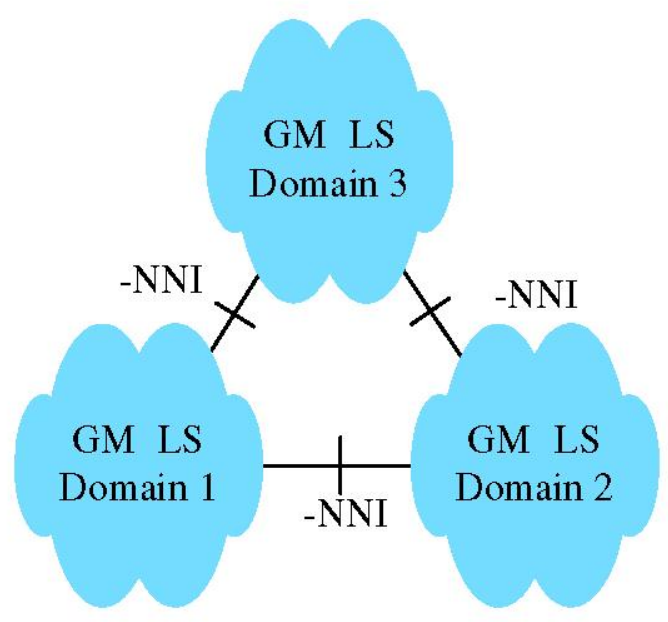

Fig. 7. Multi-domain network environment.
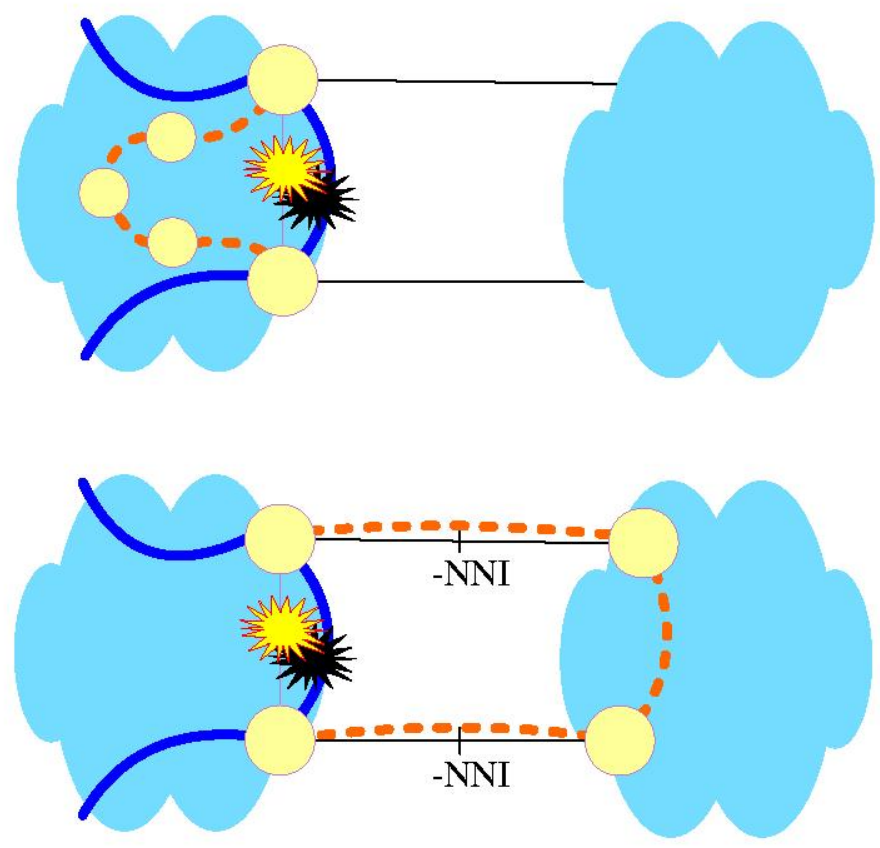

Fig. 8. Span restoration in single-domain and multi-domain environments. Situations may occur, when restoration through a neighboring network provides a more cost-efficient solution in terms of hop count or any other measure.

between GMPLS domains are specified by External Network Network Interfaces (E-NNI) in ASON [4].

The reason why topology information is not disclosed in the E-NNI is twofold. Disclosing the routing information would limit the scalability of the interconnected network by significantly increasing the routing overhead. Secondly, a network operator would not disclose his topology information, because this would expose weaknesses in his topology that might be exploited by malicious clients. Hence scalability and confidentiality are the key arguments to hide the topology information. The SV does not breach these confidentiality requirements as the information in the SV cannot be used to derive the other domains' topologies.

The lack of routing information flow across E-NNI interfaces poses a challenge in the backup route computation of span restoration. Normally, when the working path traverses several domains, the backup path is computed separately for each domain [14]. For span restoration, this will obviously cause problems when the failed span connects two domains. Even if the failed span is entirely within one domain, the shortest span restoration backup route may go through a second domain, as depicted in fig. 8 . However, finding this path requires modifications to the traditional domain-by-domain route computation. Today the use of a neighboring domain for failure recovery is not used, however, new approaches for multi-domain interoperability, as described in [15], are emerging. Increased cost efficiency obtained by using another network would be the main driver for such recovery actions.

A third issue that arises with multi-domain networks and the SV scheme is possible unfair WC distribution between 
the domains. The SV scheme provides information on the required number of WCs in the available optical paths. The overall number of WCs is useful for the destination node to choose the overall path with fewest converters. An inherent assumption in the SV scheme is that all nodes agree on the optimal choice of wavelength (the one leading to minimum wavelength conversion for the entire path). In a multi-domain environment, the view on the optimal label may differ between domains, since the choice of one label may minimize the WC usage in one domain at the expense of more conversions in the other. A malicious neighboring network might choose a path with unfair distribution of the WCs and a cost model should thus be agreed between the networks participating in the E-NNI.

The impact of the limitation imposed by the E-NNI is in the following used to define three future simulation scenarios. The scenarios differ on the amount of information that is disclosed from each network through the domain interface. In the first scenario, the reference scenario, both routing and SV information is disclosed. As such, this scenario is similar to the situation where all the nodes in question belong to the same administrative domain. The second scenario is restricted to the current implementations of the E-NNI [4]. Hence, neither routing nor SV information is passed. Finally, in the third scenario only the SV information is passed. An overview of the simulation scenarios is provided in table II.

TABLE II

MULTI-DOMAIN INFORMATION DISCLOSURE SCENARIOS

\begin{tabular}{|c|c|}
\hline Scenario & Disclosed information between domains \\
\hline Scenario 1 & Routing and SV information \\
Scenario 2 & None \\
Scenario 3 & SV information \\
\hline
\end{tabular}

Scenario 1 should be used for reference only as the scalability and the confidentiality issues related to visible topology information cancel the interest for further implementation. Scenario 2 should be evaluated to determine the impact of restricting routing and SV information. Finally, for scenario 3 , the performance compared to scenario 2 should be clarified and the inclusion of the SV information in the signalling protocols should be considered. In addition it is required to indicate measures to ensure a fair distribution of WC resources between the involved domains either by strict policies or by cost models.

\section{CONCLUSION}

We study the recovery performance of a standard (Label Set) and WC-saving (SV) label assignment scheme with and without stub-awareness in a GMPLS controlled optical network. We evaluate how these label assignment schemes affect the recovery percentage, when a single span failure is recovered using span restoration. The performance is compared under varying network load and WC availability.

Simulation results show that the SV scheme outperforms the Label Set scheme. Both schemes benefit from the stubaware modification. The modification is especially useful, if a limited number of WCs is available, and at medium load ranges.

The performance gain of the SV scheme comes at a low complexity increase. The SV object is propagated together with the Path message and does not need to be stored at the individual nodes. The stub-aware modification only has to be executed at the failure-adjacent nodes and does not affect the operation at the intermediate nodes, so the added complexity is minimal.

Furthermore, we have discussed how the SV scheme can be applied to multi-domain networks, and which methods could be introduced in the signaling messages to ensure a fair distribution of WC resources throughout the different domains.

\section{ACKNOWLEDGMENT}

This work has been partially supported by the European Commission through the Network of Excellence e-Photon/ONe+.

\section{REFERENCES}

[1] J.-P. Vasseur, M. Pickavet and P. Demeester, Network Recovery, Protec tion and Restoration of Optical, SONET-SDH, IP, and MPLS. MorganKaufmann Publishers, Elsevier, 2004.

[2] H. Zang, J. P. Jue and B. Mukherjee, "A Review of Routing and Wavelength Assignment Approaches for Wavelength-Routed Optical WDM Networks," Optical Netw. Mag., Jan. 2000.

[3] E. Mannie (ed.), "Generalized Multi-Protocol Label Switching (GMPLS) architecture," RFC 3945, Oct. 2004.

[4] International Telecommunication Union (ITU-T), "Recommendation G.8080: Architecture for the Automatically Switched Optical Network (ASON)."

[5] B. Ramamurthy and B. Mukherjee, "Wavelength conversion in WDM networking," IEEE Journal on Selected Areas in Communications, vol. 16, no. 7, pp. 1061-1073, Sept. 1998.

[6] J. Buron, S. Ruepp, and N. Andriolli, "Blocking reduction of span restoration requests in GMPLS controlled WDM optical networks," in Proc. ECOC 2006, Sept. 2006.

[7] N. Andriolli, J. Buron, S. Ruepp, F. Cugini, L. Valcarenghi and P. Castoldi, "Label Preference Schemes in GMPLS Controlled Networks," IEEE Comm. Lett., vol. 12, no. 10, pp. 849-851, Dec. 2006.

[8] L. Berger (ed.), "Generalized Multi-Protocol Label Switching (GMPLS) signaling functional description," RFC 3471, Jan. 2003.

[9] S. Ruepp, J. Buron and N. Andriolli, "Increasing Restorability for Localto-Egress Restoration in GMPLS Controlled Networks with Limited Wavelength Conversion," in Proc. ICCS 2006., Oct. 2006.

[10] - "Wavelength-Converter Saving Span Restoration in GMPLS Controlled WDM Optical Networks," in Proc. Conference of Optical Internet, COIN 2006, July 2006.

[11] M. Miyao and H. Saito, "Optimal Design and Evaluation of Survivable WDM Transport Networks," IEEE Joumal on Selected Areas in Communications, vol. 16, no. 7, pp. 1190-1199, 1998.

[12] OPNET Technologies, Inc., http://www.opnet.com.

[13] A. Farrel (ed.), "Crankback Signaling Extensions for MPLS and GMPLS RSVP-TE," draft-ietf-ccamp-crankback-06.txt, IETF Draft, Jan. 2007, work in progress.

[14] A. Farrel and I. Bryskin, GMPLS Achitecture and Applications. Morgan Kaufmann, Elsevier, 2006.

[15] H.-M. Foisel, J. Spaeth, C. Cavazzoni, H. Wessing and M. Popov, "MUPBED - interworking challenges in a multi-domain and multitechnology network environment," Proc. of Optical Network Design and Modeling (ONDM 2007), Athens, Greece, pp. 269-278, May 2007. 